\title{
Health Care Utilization and Economic Burdens of Hemochromatosis in the United States: A Population-Based Claims Study
}

\author{
Mohamed I. Elsaid, MPH, ALM, LEED-GA; Tina John, MD, MPH; You Li, BS; Sobha Koduru, MD; \\ Saima Z. Ali, MD; Carolyn Catalano, APN; Navaneeth Narayanan, PharmD, BCPS; \\ and Vinod K. Rustgi, MD, MBA
}

\begin{abstract}
BACKGROUND: Little is known about the health care burden of hemochromatosis in the United States, despite its increased morbidity and mortality due to associated advanced liver diseases.
\end{abstract}

OBJECTIVE: To evaluate the health care utilization and economic burdens of hemochromatosis in the United States using real-world claims data.

METHODS: We performed a case-control analysis of adult participants in the Truven Health MarketScan Commercial Claims database from 2010 to 2015. 37,092 hemochromatosis cases were matched $1: 1$ by demographics and comorbidities to hemochromatosis-free controls with chronic liver disease using propensity scores. Total and service-specific health care parameters were quantified for the 12 months following versus the 12 months before the first date of hemochromatosis diagnosis and over the 12 months following a randomly selected date for controls. Incremental differences in health care burdens between cases and controls were examined using Wilcoxon signed rank tests and McNemar tests for continuous and dichotomous measures, respectively. Adjusted multivariable regression analyses using generalized linear models were used to compare the health care burdens for cases with controls.

RESULTS: In comparison with the year before, the 12 months following first hemochromatosis diagnoses had a higher total number of claims per patient (34.37 vs. $29.99 ; P<0.0001$ ) and an increase in the per-patient total health care costs $(\$ 20,023$ vs. $\$ 16,905 ; P<0.0001)$. After hemochromatosis diagnosis, health care costs were $2 \%, 8 \%, 23 \%$, and $43 \%$ higher for inpatient admissions, emergency department visits, outpatient visits, and pharmaceutical prescriptions, compared respectively with the 12 months before diagnosis. In the 12 months following the index date, hemochromatosis cases incurred $\$ 2,732$ more in total unadjusted costs compared with controls. Compared with controls, cases had adjusted incident rate ratio (IRR) $1.26(95 \% \mathrm{Cl}=1.30-1.77)$ times the total number of claims (IRR=1.40, $95 \% \mathrm{Cl}=1.38-1.43)$ more outpatient visits and $\mathrm{IRR}=1.10(95 \% \mathrm{Cl}=1.08-1.11)$ excess pharmaceutical claims. Compared with controls, cases had significantly higher adjusted mean health care costs for inpatient services $(\$ 6,484$ vs. $\$ 7,854)$, outpatient services $(\$ 7,032$ vs. $\$ 11,005)$, and pharmaceutical claims $(\$ 2,520$ vs. $\$ 2,822$; all $P$ values $<0.05)$. The annual health care costs among type 2 diabetes, hypertension, arthritis, and chronic kidney disease (CKD) patients with hemochromatosis were $\$ 6,968, \$ 7,424$, $\$ 2,967$, and $\$ 43,847$, respectively, higher than type 2 diabetes, hypertension, arthritis, and CKD patients without hemochromatosis $(P<0.0001)$.

CONCLUSIONS: Hemochromatosis in the United States is associated with significant health care utilization and economic burdens driven by outpatient visits, pharmaceutical claims, and a high number of comorbidities

J Manag Care Spec Pharm. 2019;25(12):1377-86

Copyright $\odot 2019$, Academy of Managed Care Pharmacy. All rights reserved.

\section{What is already known about this subject}

Hemochromatosis increases the risk of tissue damage and multiorgan failure due to excess iron accumulations.

Quantifying the burden of hemochromatosis is essential in assessing the cost-effectiveness of intervention strategies such as screening.

\section{What this study adds}

This study used real-life data to conduct a propensity scorematched case-control analysis to quantify the annual health care resource utilization and economic burdens of hemochromatosis in the United States.

Study findings demonstrate that a new hemochromatosis diagnosis results in excess annual health care utilization of 4.38 claims per patient and an additional $\$ 3,118$ in per-patient yearly health care cost.

$\mathrm{H}$ ereditary hemochromatosis is a common autosomal recessive disorder, affecting 1 in 200-250 Caucasians of Northern European origin. ${ }^{1}$ An estimated $90 \%$ of hemochromatosis occurrence is associated with a homozygous C282Y mutation in the HFE gene. ${ }^{2}$ This mutation affects the ability of the HFE protein to regulate hepcidin synthesis, a key mechanism in iron homeostasis. ${ }^{2-5}$ As such, hepcidin deficiencies result in parenchymal iron excess due to elevated hepatic uptake of non-transferrin bound iron. ${ }^{5}$ Progressive accumulations of this excess iron culminate in tissue damage and multiorgan failure.,

Initial signs and symptoms are nonspecific, which makes the diagnosis of hemochromatosis challenging at times. Accordingly, hemochromatosis is often diagnosed late in the disease process, when irreversible organ damage has been well established due to progressive iron accumulation. ${ }^{7}$ The liver is particularly affected, and untreated hepatic iron accumulation can result in hepatomegaly, cirrhosis complicated by liver failure, hepatocellular carcinoma (HCC) or HCC in the absence of cirrhosis. ${ }^{8-10}$ Studies have also identified hepatic iron content as an independent predictor of liver fibrosis. ${ }^{11}$

Early diagnosis and management are essential in preventing irreversible organ damage and increasing the survival of hemochromatosis patients. ${ }^{12,13}$ As a result, screening of those 
at risk has been proposed as an effective method to increase early detection, reducing the health care burden of hemochromatosis and its associated comorbidities., ${ }^{914}$ However, lack of robust real-world data has been cited as a barrier in evaluating the cost-effectiveness of such screening programs. ${ }^{15,16}$ Furthermore, the cost of health care continues to rise in the United States and is projected to reach $20 \%$ of the economy by $2024 .{ }^{17}$ Consequently, conducting a full evaluation of the health care burden of hemochromatosis is an essential task that could aid in policy assessments and resource allocations.

To date, only 1 study has evaluated the economic and health care burdens of hemochromatosis, using self-reported cost data on 157 patients in Australia. ${ }^{12}$ The study reported a significant increase in the economic burden of hemochromatosis as a function of disease severity. Namely, the total economic burdens in the mildest and most severe forms of hemochromatosis were Australian dollar (AUD) 1,431 and AUD 11,882, respectively. The total societal costs of hemochromatosis were 3 times higher in symptomatic versus asymptomatic hemochromatosis patients (AUD 10,030 vs. AUD 3,701). The study concluded that increased economic burden in symptomatic patients was attributed to higher health care costs. Findings from this study, however, do not represent the case for the economic and health care utilization burdens of hemochromatosis in the United States.

The economic and health care utilization burdens of hemochromatosis in the United States remain unevaluated despite its prevalence and the increased morbidity and mortality risks due to associated end-stage liver disease. Health insurance claims are real-life data that can be used to estimate health care costs and utilization for hemochromatosis patients, while adjusting for individual demographics and disease characteristics. In this real-time population study, we used commercial insurance data to conduct a propensity score-matched case-control analysis to quantify the health care resource utilization and economic burdens of hemochromatosis in the United States. The evaluation of such burdens is essential in assessing the cost-effectiveness of intervention strategies, such as screening, informing policymakers, and enhancing resource allocations efforts.

\section{Methods}

\section{Study Design and Data Source}

This study is a case-control analysis of the Truven Health MarketScan Commercial Claims (MSCC) databases for the period January 1, 2010-December 31, 2015.

To account for the continuous enrollment requirements of 12 months before and after the index date, we defined an index period for both cases and controls between January 1 , 2011, and December 31, 2014. The claims data represent health care records from government and public organizations, large employers, and health plans from approximately 350 payers annually. The MSCC databases include longitudinal individual-specific linked data for health insurance claims across inpatient, outpatient, and prescription drug services. The total gross cost of care provided in the MSCC denotes the amount eligible for payment before applying coordination of benefits, deductibles, and copayments. All MSCC records are deidentified data that are compliant with all U.S. patient confidentiality requirements, including the Health Insurance Portability and Accountability Act (HIPAA) of 1996. The Internal Review Board of Rutgers Robert Wood Johnson Medical School approved the study protocol.

\section{Study Participants}

Records from inpatient admissions and outpatient services were used to classify participants as either with or without chronic liver disease (CLD). The CLD cohort was identified as 1 primary or secondary inpatient admission or outpatient service diagnosis of International Classification of Disease, Ninth Revision, Clinical Modification (ICD-9-CM) codes for hemochromatosis, liver transplant, HCC, cirrhosis, hepatitis C virus (HCV), chronic hepatitis B (CHB), other hepatitis (i.e., autoimmune hepatitis or hepatitis E virus), primary biliary cirrhosis (PBC), primary sclerosing cholangitis (PSC), alcoholic fatty liver, or nonalcoholic fatty liver disease (NAFLD). From the CLD cohort, we identified patients with hemochromatosis using ICD-9-CM codes 275.0, 275.01, 275.02, 275.03, or 275.09. In turn, we identified potential cases as participants with hemochromatosis, while potential controls were patients with any CLD and without hemochromatosis diagnosis.

We defined an index date for each potential case as the earliest date of hemochromatosis diagnosis. For potential controls, we randomly selected an index date from the list of all claims for CLD, starting with the earliest date of liver transplant, HCC, cirrhosis, HCV, CHB, other hepatitis, PBC, PSC, alcoholic fatty liver, or NAFLD. The random selection of an index date for controls allowed for the burden of hemochromatosis to be compared with that of the average CLD burden through all stages of the disease spectrums.

A baseline period was defined for all participants as the 12 months before the index date. The study follow-up periods represented the 12 months following each participant's index date. Only adult (aged 18+ years) participants with continuous enrollments of at least 12 months before and 12 months following the index date were considered for this study.

\section{Patient Characteristics and Comorbidity Profile}

Baseline demographics including age, gender, the region of residence, and the type of health insurance plan were obtained from the index date. A comorbidity profile was measured for each participant during the baseline periods using ICD-9-CM codes obtained from inpatient admissions and outpatient services. The profile included hemochromatosis risk factors such as type 2 diabetes mellitus (T2DM), arthritis, alcohol abuse or dependence (AAD), and myocardial infarction (MI). ${ }^{18}$ 


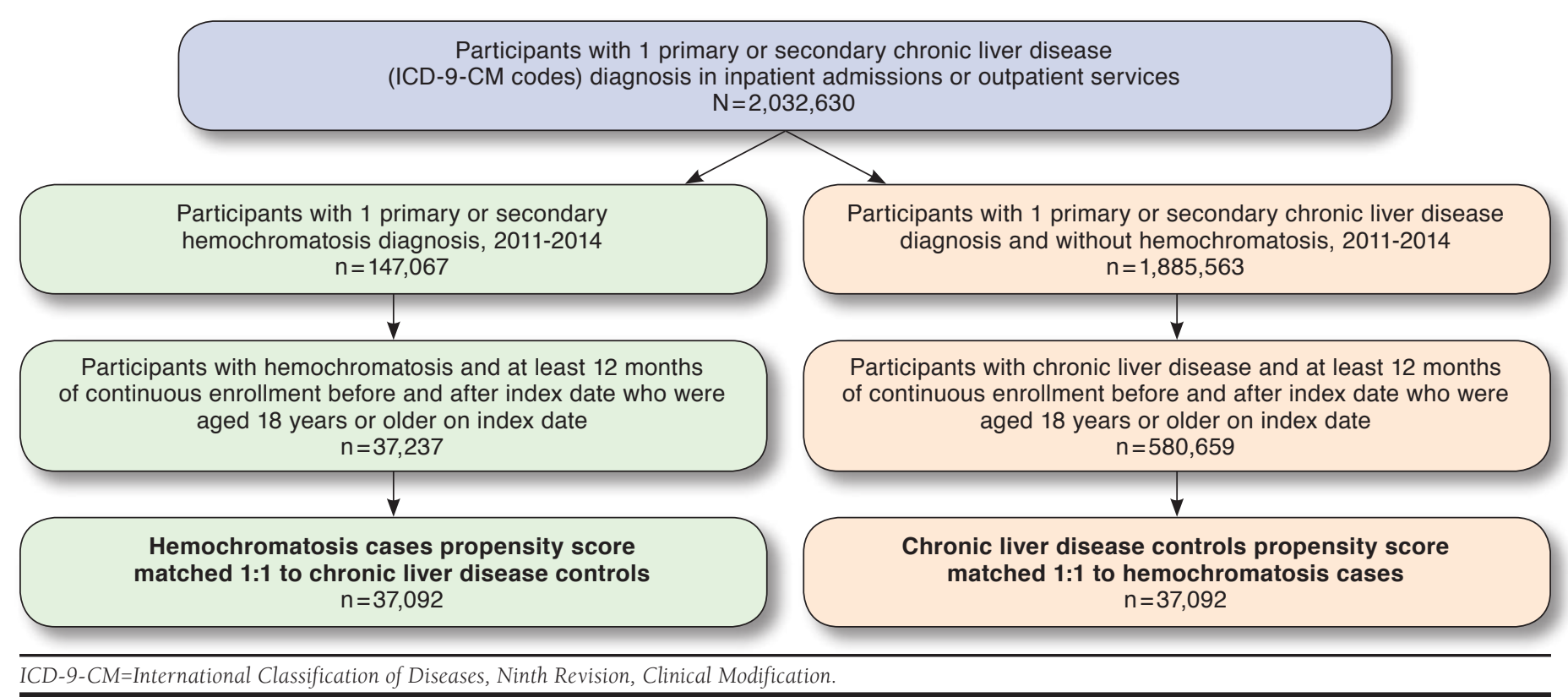

We also included data on hypertension, CLD, chronic kidney disease (CKD), heart failure, and a total weighted Charlson Comorbidity Index (CCI) score using the enhanced ICD-9-CM algorithm provided by Quan et al. (2005). ${ }^{19}$

\section{Matching Procedure}

We used propensity score matching to ensure the comparability in the distributions of all observed baseline demographics and comorbidity profiles between cases and controls. A multivariate logistic regression model, with hemochromatosis status as the outcome, that included age; gender; the region of residence; type of health insurance; CCI category (excluding myocardial infarction, rheumatic disease, diabetes without chronic complications, and diabetes with chronic complications); T2DM; arthritis; AAD; and MI was used to estimate a propensity score for each participant. In turn, hemochromatosis cases were matched 1:1 to hemochromatosis-free controls using the greedy algorithm. ${ }^{20}$

\section{Health Care Utilization and Costs}

Measures of health care utilization included the average number of claims per patient per year for inpatient admissions, emergency department (ED) visits, outpatient visits, and pharmaceutical prescriptions. Cost estimates represented the average per-patient expenditures related to inpatient admissions, ED visits, outpatient visits, and pharmaceutical prescriptions. We also estimated the prevalence of having at least one of the following: inpatient admission, outpatient visit, and ED visit for all participants. The average lengths of inpatient stay per patient were also calculated for all cases and controls. Only emergency services that did not result in inpatient admission were classified as ED visits.

Health care utilization and cost parameters were aggregated over the 12 months before and after the first day of hemochromatosis diagnosis to assess the burden associated with a new diagnosis. Also, we quantified all use and cost parameters over the 12 months following the randomly selected index date for controls to compare the burden of hemochromatosis with that of matched CLD controls.

In a subanalysis, we quantified the age group-specific health care cost comparisons between matched cases and controls for all 5 expenditure variables. We also conducted a subgroup analysis to determine the differences in health care costs between matched pairs of cases and controls relative to comorbidities associated with hemochromatosis. All costs estimates were adjusted to 2017 U.S. dollars, using the medical care commodities component of the Consumer Price Index.

\section{Statistical Analysis}

We compared baseline characteristics and comorbidity profiles for those with and without hemochromatosis before and after matching using the standardized differences of means for continuous variables and the standardized differences of proportions for categorical variables. We used standardized difference cutoffs of $0.2,0.5$, and 0.8 to indicate small, medium, 
TABLE 1 Baseline Characteristics of the Study Sample of Adult Participants with Chronic Liver Disease by Hemochromatosis Status Before and After Matchinga

\begin{tabular}{|c|c|c|c|c|c|c|}
\hline \multirow[b]{2}{*}{ Patient Characteristics } & \multicolumn{3}{|c|}{ Unmatched } & \multicolumn{3}{|c|}{ Matched ${ }^{b}$} \\
\hline & $\begin{array}{l}\text { Hemochromatosis } \\
\quad(\mathbf{n}=37,237)\end{array}$ & $\begin{array}{c}\text { No } \\
\text { Hemochromatosis } \\
(\mathrm{n}=580,659)\end{array}$ & \multirow{2}{*}{\begin{tabular}{|c|}
$\begin{array}{c}\text { Standardized } \\
\text { Difference }^{c}\end{array}$ \\
0.1574 \\
\end{tabular}} & $\begin{array}{l}\text { Hemochromatosis } \\
\qquad(\mathbf{n}=37,092)\end{array}$ & $\begin{array}{c}\text { No } \\
\text { Hemochromatosis } \\
(\mathrm{n}=37,092)\end{array}$ & \multirow{2}{*}{$\begin{array}{c}\begin{array}{c}\text { Standardized } \\
\text { Difference }^{c}\end{array} \\
0.0065\end{array}$} \\
\hline Age, mean (SD) & $47.09(11.32)$ & $48.82(10.77)$ & & 47.09 (11.31) & $47.17(11.27)$ & \\
\hline Age group, years, n (\%) & & & 0.1506 & & & 0.0069 \\
\hline $18-34$ & $5,623 \quad(15.1)$ & $68,387 \quad(11.8)$ & & $(15.1)$ & $(14.8)$ & \\
\hline $35-44$ & $7,856 \quad(21.1)$ & $108,384 \quad(18.7)$ & & $(21.1)$ & $(21.1)$ & \\
\hline $45-54$ & $12,251 \quad(32.9)$ & $188,462 \quad(32.5)$ & & 12,204 & 12,224 & \\
\hline $55+$ & $11,507 \quad(30.9)$ & $215,426 \quad(37.1)$ & & 11,469 & 11,529 & \\
\hline Gender, n (\%) & & & 0.0179 & & & 0.0023 \\
\hline Male & $17,337 \quad(46.6)$ & $275,530 \quad(47.5)$ & & 17,271 & $17,228 \quad(46.5)$ & \\
\hline Female & $19,900 \quad(53.4)$ & $305,129 \quad(52.6)$ & & 19,821 & 19,864 & \\
\hline Region of residence, n (\%) & & & 0.1417 & & & 0.0055 \\
\hline Northeast & $9,329 \quad(25.1)$ & $126,950 \quad(21.7)$ & & $(25.0)$ & $(25.1)$ & \\
\hline North Central & $7,325 \quad(19.7)$ & $103,548 \quad(17.8)$ & & $(19.7)$ & $(19.7)$ & \\
\hline South & $12,229 \quad(32.8)$ & $229,570 \quad(39.5)$ & & 12,180 & 12,225 & \\
\hline West & $7,542 \quad(20.3)$ & $109,865 \quad(18.9)$ & & $(20.3)$ & $(20.2)$ & \\
\hline Unknown & $812 \quad(2.2)$ & $10,726 \quad(1.9)$ & & $(2.2)$ & $(2.1)$ & \\
\hline Type of health insurance, $\mathrm{n}(\%)$ & & & 0.1113 & & & 0.0155 \\
\hline Unknown & 1,277 & 20,366 & & 1,265 & $(3.36)$ & \\
\hline Comprehensive & $779 \quad(2.1)$ & 16,647 & & $(2.1)$ & $(2.1)$ & \\
\hline Exclusive provider organization & $764 \quad(2.1)$ & $9,871 \quad(1.7)$ & & $(2.1)$ & $(2.0)$ & \\
\hline Health maintenance organization & $3,881 \quad(10.4)$ & $75,695 \quad(13.0)$ & & $(10.4)$ & $(10.5)$ & \\
\hline Non-capitated point-of-service & $2,869 \quad(7.7)$ & $44,680 \quad(7.7)$ & & 2,861 & 2,829 & \\
\hline Preferred provider organization & $23,815 \quad(64.0)$ & $354,427 \quad(61.0)$ & & 23,752 & 23,859 & \\
\hline Point-of-service with capitation & $169 \quad(0.5)$ & $2,503 \quad(0.4)$ & & $(0.5)$ & $(0.4)$ & \\
\hline Consumer-driven health plan & $2,182 \quad(5.9)$ & 37,395 & & 2,168 & 2,179 & \\
\hline High-deductible health plan & 1,501 & 19,075 & & 1,490 & 1,439 & \\
\hline \multicolumn{7}{|l|}{ Comorbidity profiled } \\
\hline $\begin{array}{l}\text { Charlson Comorbidity Index } \\
\text { score, mean (SD) }\end{array}$ & $0.69(1.36)$ & $1.12(1.82)$ & 0.2658 & $(1.36)$ & $(1.46)$ & 0.0125 \\
\hline $\begin{array}{l}\text { Charlson Comorbidity Index } \\
\text { score, } \mathrm{n}(\%)\end{array}$ & & & 0.2975 & & & 0.0049 \\
\hline 0 & $24,372 \quad(65.5)$ & $301,235 \quad(51.9)$ & & 24,281 & 24,305 & \\
\hline 1 & $7,033 \quad(18.9)$ & $136,867 \quad(23.6)$ & & $(18.8)$ & $(19.0)$ & \\
\hline 2 & $2,848 \quad(7.6)$ & $57,624 \quad(9.9)$ & & 2,838 & 2,807 & \\
\hline 3 & $1,423 \quad(3.8)$ & 38,098 & & 1,418 & 1,421 & \\
\hline $4+$ & $1,561 \quad(4.2)$ & $46,835 \quad(8.1)$ & & 1,556 & 1,531 & \\
\hline Type 2 diabetes, $\mathrm{n}(\%)$ & $5,453 \quad(14.6)$ & $139,698 \quad(24.1)$ & 0.2400 & $(14.6)$ & $(14.7)$ & 0.0015 \\
\hline Arthritis, n (\%) & $15,483 \quad(41.6)$ & $264,306 \quad(45.5)$ & 0.0795 & 15,415 & 15,430 & 0.0008 \\
\hline Alcohol abuse or dependence, $\mathrm{n}(\%)$ & $199 \quad(0.5)$ & $7,970 \quad(1.4)$ & 0.0863 & $(0.5)$ & $(0.5)$ & 0.0107 \\
\hline Myocardial infarction, $\mathrm{n}(\%)$ & $(0.8)$ & 8,893 & 0.0734 & $(0.8)$ & $(0.8)$ & 0.0080 \\
\hline \multicolumn{7}{|c|}{$\begin{array}{l}\text { aAll demographics data were obtained on index date (first diagnosis date of hemochromatosis for cases and a randomly selected date from all claim records for controls). } \\
\text { bHemochromatosis cases and hemochromatosis-free controls were matched 1:1 using propensity score. The logistic regression model used to estimate propensity scores } \\
\text { included age; region of residence; gender; type of health insurance; Charlson Comorbidity Index score (excluding myocardial infarction, rheumatic disease, diabetes without } \\
\text { chronic complications, and diabetes with chronic complications); myocardial infarction; type } 2 \text { diabetes; arthritis; and alcohol abuse or dependence. } \\
\text { cDifference in means or proportions divided by standard error. Imbalance between the } 2 \text { groups was defined as absolute value greater than 0.10; smaller values indicated } \\
\text { better balance. } \\
\text { dEstimated during the } 12 \text { months before the index date. } \\
\text { SD=standard deviation. }\end{array}$} \\
\hline
\end{tabular}

and large differences between means and proportions of the 2 comparison groups. ${ }^{21,22}$ Wilcoxon signed rank tests were used to compare all continuous measures of health care costs and utilization in the before and after diagnosis evaluations and in the cases versus controls analyses, while McNemar tests were used to examine all dichotomous variables. 
TABLE 2 Health Care Resource Utilization for Hemochromatosis Patients During the 12 Months Before Versus 12 Months After the First Diagnosis Date, 2011-2014

\begin{tabular}{|c|c|c|c|}
\hline Health Care Utilization & $\begin{array}{c}\text { Prediagnosis } \\
(\mathrm{n}=37,092)\end{array}$ & $\begin{array}{l}\text { Postdiagnosis } \\
(\mathrm{n}=37,092)\end{array}$ & $P$ Value ${ }^{\mathrm{a}}$ \\
\hline \multicolumn{4}{|l|}{ Total number of claims } \\
\hline Mean (SD) & $29.99 \quad(30.50)$ & $34.37 \quad(33.67)$ & $<0.0001$ \\
\hline Median (25th, 75 th percentile) & $21 \quad(10,39)$ & $25 \quad(13,45)$ & \\
\hline \multicolumn{4}{|l|}{ Inpatient admissions } \\
\hline Prevalence of at least 1 visit, $\mathrm{n}(\%)$ & $(10.8)$ & $(10.6)$ & 0.2328 \\
\hline \multicolumn{4}{|l|}{ Number of admissions } \\
\hline Mean (SD) & $0.80 \quad(5.12)$ & $(5.63)$ & 0.0052 \\
\hline Median (25th, 75th percentile) & $(0,0)$ & $(0,0)$ & \\
\hline \multicolumn{4}{|l|}{ Total length of stay, days } \\
\hline Mean (SD) & $(5.85)$ & $(5.71)$ & 0.9531 \\
\hline Median (25th, 75th percentile) & $(0,0)$ & $(0,0)$ & \\
\hline \multicolumn{4}{|l|}{ Emergency department visits } \\
\hline Prevalence of at least 1 visit, $\mathrm{n}(\%)$ & $(19.7)$ & $(19.3)$ & 0.1162 \\
\hline \multicolumn{4}{|l|}{ Number of visits } \\
\hline Mean (SD) & $0.34 \quad(1.06)$ & $(1.09)$ & 0.7223 \\
\hline Median (25th, 75th percentile) & $(0,0)$ & $(0,0)$ & \\
\hline \multicolumn{4}{|l|}{ Outpatient visits } \\
\hline Prevalence of at least 1 visit, $\mathrm{n}(\%)$ & 37,080 & 37,087 & 0.1435 \\
\hline \multicolumn{4}{|l|}{ Number of visits } \\
\hline Mean (SD) & $17.19 \quad(19.31)$ & $20.07 \quad(22.26)$ & $<0.0001$ \\
\hline Median (25th, 75th percentile) & $(6,21)$ & $(7,25)$ & \\
\hline \multicolumn{4}{|l|}{ Pharmaceutical claims } \\
\hline \multicolumn{4}{|l|}{ Number of claims } \\
\hline Mean (SD) & $11.66 \quad(14.35)$ & $13.10 \quad(15.30)$ & $<0.0001$ \\
\hline Median (25th, 75 th percentile) & $(0,17)$ & $9 \quad(1,19)$ & \\
\hline
\end{tabular}

To ensure the robustness of our primary results, we conducted multivariable regression analyses using generalized linear models (GLM) with negative binomial distributions for health care utilization rates and gamma distributions for cost estimates. We used generalized estimation equations to account for the correlation between matched cases and controls. The results of the GLM analyses represented adjusted incident rate ratios (IRRs) for utilization comparisons between hemochromatosis cases and hemochromatosis-free controls. We also used GLM models to estimate the predicted adjusted mean costs for cases and controls. We quantified 95\% confidence intervals (CIs) and $P$ values for all health care cost and utilization parameters. A $P<0.05$ was considered statistically significant. SAS software version 9.4 was used to perform all data analyses (SAS Institute, Cary, NC).

\section{Results}

Our study sample included 617,896 adult participants in the MSCC database who met the inclusion criteria of continuous enrollment during the baseline period and for 12 months following the index dates (Figure 1). Compared with CLD patients without hemochromatosis, those with hemochromatosis were younger (48.82 vs. 47.09 years; $P<0.0001$ ) with a slightly lower proportion of males (47.6\% vs. $46.6 \%$; $P<0.0008)$ and a smaller weighted CCI score (1.12 vs. 0.69; $P<0.0001$; Table 1).

Hemochromatosis patients also had lower prevalence of T2DM (14.6\% vs. $24.1 \%$ ), arthritis (41.6\% vs. $45.5 \%)$, AAD ( $0.5 \%$ vs. $1.4 \%)$, and MI (0.8\% vs. $1.5 \%)$ compared with hemochromatosis-free participants with CLD $(P<0.0001)$. Of all 37,237 hemochromatosis patients who met the inclusion criteria, we matched 37,092 hemochromatosis cases 1:1 using propensity scores to hemochromatosis-free CLD controls. Overall distribution balances between matched cases and controls were obtained for all baseline characteristics and comorbidity profiles (Table 1).

Among cases, the total number of claims was significantly higher in the 12 months following the first hemochromatosis diagnosis compared with the 12 months before the first diagnosis (34.37 vs. 29.99; $P<0.0001$ ). Similarly, in the 12 months 


\section{FIGURE 2 Annual Per-Patient Health Care Costs in the 12 Months Before Versus 12 Months After} First Hemochromatosis Diagnosis, 2011-2014

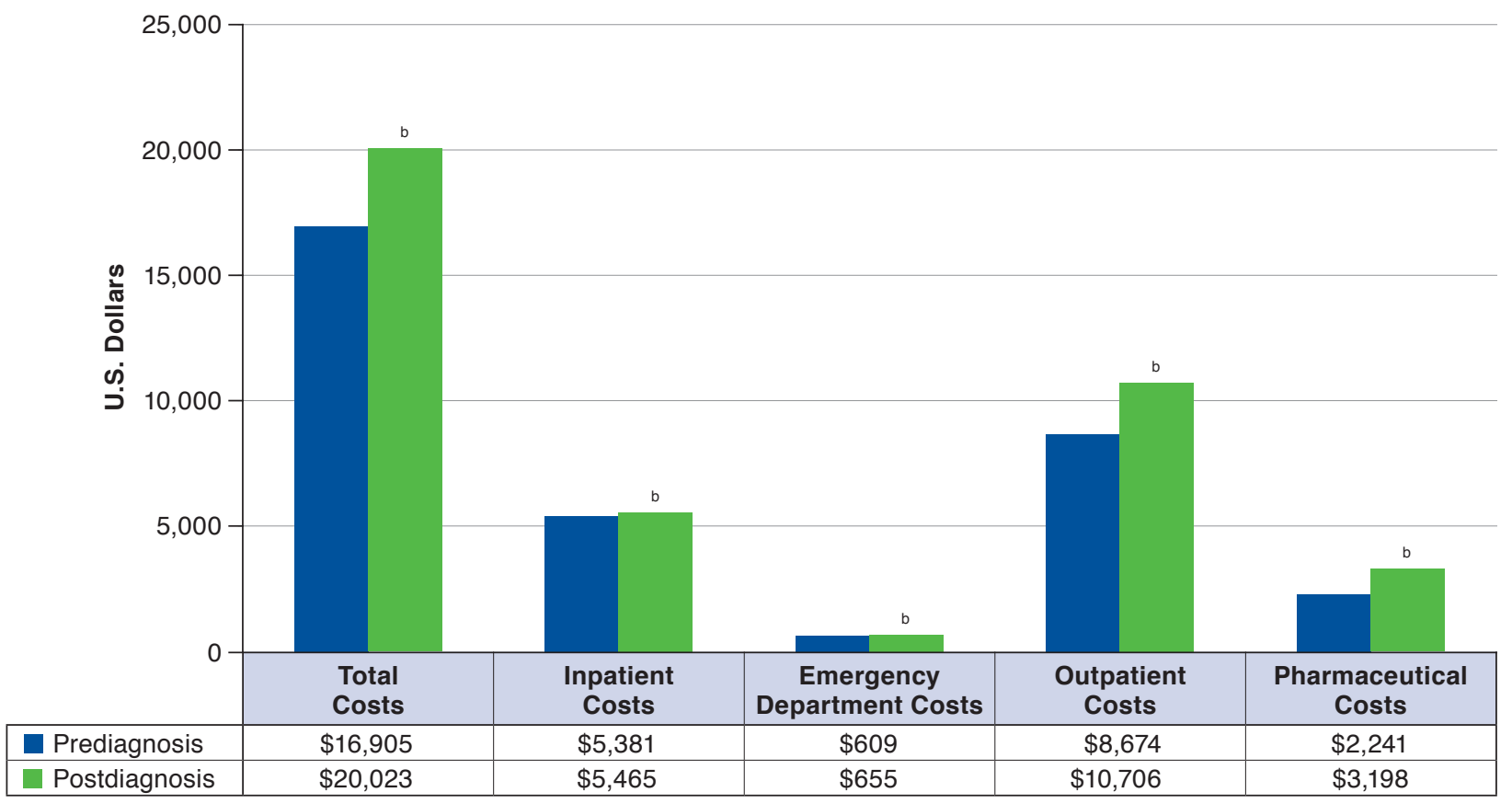

aAll costs were adjusted to 2017 U.S. dollars using the medical care component of the Consumer Price Index

${ }^{b}$ Wilcoxon signed rank test $P$ value $<0.05$ for health care cost comparisons before versus after first hemochromatosis diagnosis.

after versus the 12 months before the first diagnoses, new hemochromatosis diagnosis was associated with 2.88 (95\% $\mathrm{CI}=2.71-3.06)$ higher per-patient outpatient visits and 1.44 (95\% CI $=1.36-1.52)$ additional per-patient pharmaceutical claims. In the year following the first diagnosis, hemochromatosis patients had more inpatient admissions (0.86 vs. 0.80; $P<0.0052$ ) when compared with the year before first diagnosis. The proportions of patients with at least 1 inpatient admission or at least $1 \mathrm{ED}$ visit were not statistically different in the first hemochromatosis diagnoses pre- versus postperiods (Table 2).

In the year following the first diagnosis, hemochromatosis cases incurred $\$ 3,118$ (95\% CI $=\$ 2,519-\$ 3,716)$ higher per-patient health care cost compared with the cost in the prediagnosis year. The incremental difference in annual health care cost after versus before first hemochromatosis diagnosis was due to $\$ 84(2.7 \%)$ from inpatient costs, $\$ 46$ (1.5\%) from ED costs, $\$ 2,032$ (65.2\%) from cost of outpatient visits, and $\$ 957$ (30.7\%) from prescription costs. The increase in total perpatient cost after hemochromatosis diagnoses was the result of $2 \%, 8 \%, 23 \%$, and $43 \%$ higher expenditures related to inpatient admissions, ED visits, outpatient visits, and pharmaceutical prescriptions when compared respectively to the cost in the 12 months before hemochromatosis diagnosis (Figure 2).
The differences between cases and controls in total unadjusted health care cost during the 12 months following the index date were $\$ 2,433(95 \% \quad C I=\$ 1,700-\$ 3,165)$ higher among all participants irrespective of age. Aside from patients aged 18-34 years, hemochromatosis cases had significantly higher unadjusted annual costs when compared with CLD controls. The overall cost differences between cases and controls increased with age (Figure 3). The highest difference of $\$ 5,419$ (95\% CI $=\$ 3,851-\$ 6,988)$ in annual health care cost was observed between cases and controls in patients aged 55 years or older. The annual costs related to outpatient visits were higher for cases versus controls in all age groups $(P<0.0001)$. Only hemochromatosis patients aged 55 years or older incurred significantly higher costs related to inpatient admissions when compared with controls (data not shown).

The results of the adjusted GLM models yielded similar health care utilization and cost estimates relative to those obtained from the unadjusted analyses. When compared with controls in the 12 months following the index date, cases had an IRR of 1.26 (95\% CI=1.24-1.27) times the total number of claims per patient; an IRR of 1.40 (95\% CI $=1.38-0.43)$ additional outpatient visits per patient; and an IRR of 1.10 (95\% $\mathrm{CI}=1.08-1.11)$ excess in pharmaceutical claims per patient 

Cases and Matched Chronic Liver Disease Controls Without Hemochromatosis by Age Group (Years)a,b

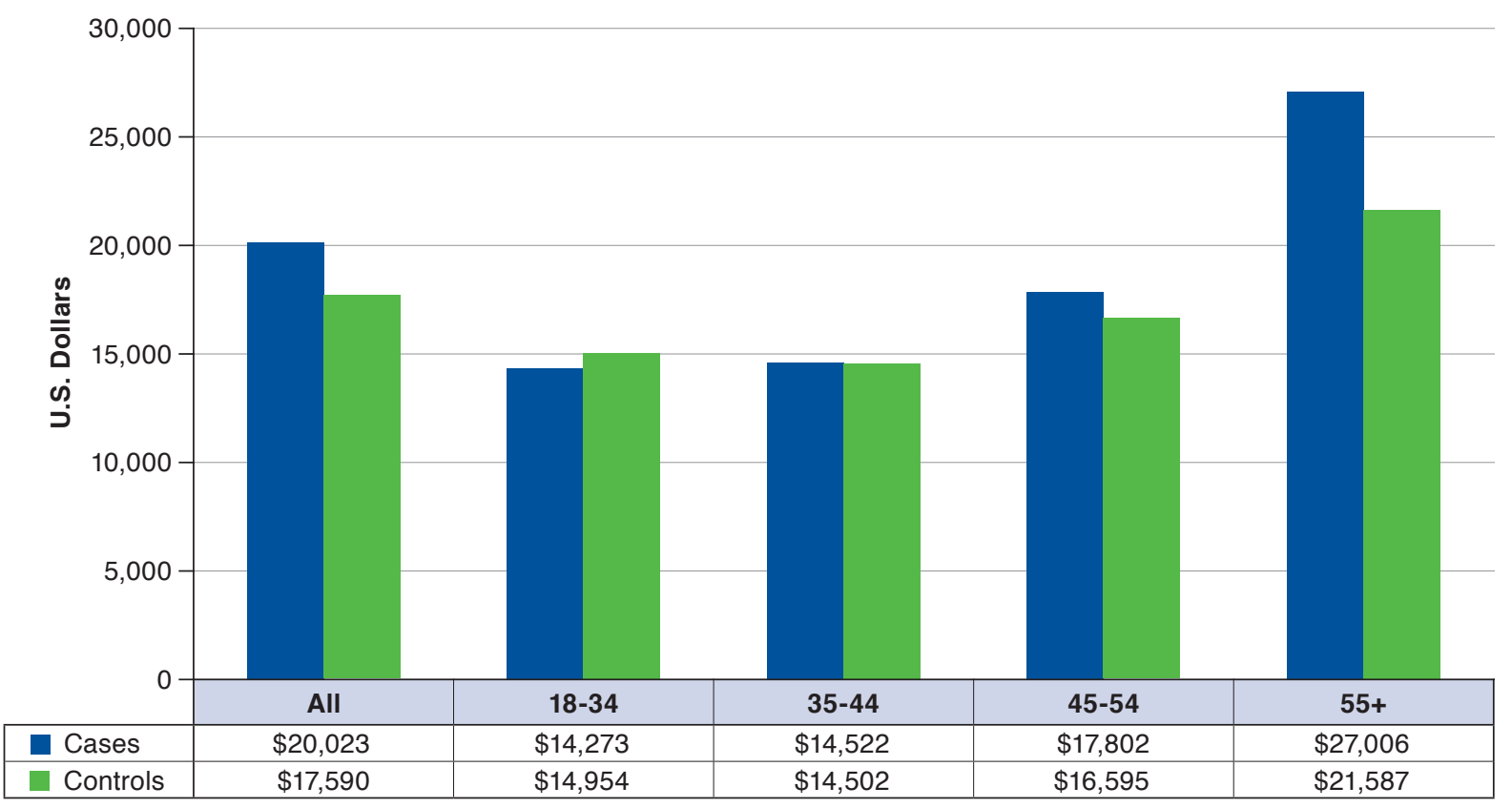

aAll Wilcoxon signed rank test $P$ values were $<0.05$ for total health care cost comparisons between cases and controls.

bAll costs were adjusted to 2017 U.S. dollars using the medical care component of the Consumer Price Index.

(data not shown). The number of ED visits for cases was an IRR of 0.84 (95\% CI $=0.80-0.88$ ) times that of hemochromatosisfree controls. No significant differences in IRR between cases and controls were observed for the number of inpatient visits.

The adjusted mean health care cost in the 12 months following the index date was $\$ 24,312(95 \% \mathrm{CI}=\$ 22,033-\$ 26,826)$ for hemochromatosis cases and $\$ 18,116$ (95\% CI $=\$ 16,359$ $\$ 20,061)$ for controls with CLD and without hemochromatosis. Compared with controls, cases had significantly higher adjusted mean health care costs for inpatient services ( $\$ 6,484$ vs. $\$ 7,854)$, outpatient services $(\$ 7,032$ vs. $\$ 11,005)$, and pharmaceutical claims $(\$ 2,520$ vs. $\$ 2,822 ; P<0.05)$.

Among those with CCI scores of 2,3 , and 4 (and above), the costs for hemochromatosis cases were $\$ 6,184, \$ 12,328$, and $\$ 31,395$, respectively, higher when compared with controls in the year following the index date (Appendix, available in online article). In the year following the index date, cases with hemochromatosis and T2DM incurred \$6,968 (95\% $\mathrm{CI}=\$ 4,477-\$ 9,459)$ higher costs than controls with T2DM and without hemochromatosis. The annual costs among hypertension, CLD, CKD, arthritis, and heart failure patients with hemochromatosis were $\$ 7,424, \$ 16,634, \$ 43,847, \$ 2,967$, and $\$ 54,047$, respectively, higher than hypertension, CLD, CKD, arthritis, and heart failure patients without hemochromatosis.

\section{Discussion}

To our knowledge, this is the first study to use real-world claims data to estimate the health care utilization and cost burdens related to hemochromatosis in the United States. Our findings demonstrate that a new hemochromatosis diagnosis results in excess annual health care utilization of 4.38 claims per patient and an additional $\$ 3,118$ in per-patient annual health care cost. We also show that hemochromatosis cost $\$ 2,433$ annually more per patient when compared with CLD controls with the same demographics and comorbidity profiles. Those findings were confirmed by the results of the adjusted analyses.

Per annual patient cost of hemochromatosis was highest among patients aged 55 years or older, and the incremental differences in yearly costs between those with versus without hemochromatosis increased with age. In the comorbidityspecific analysis, having both hemochromatosis and T2DM incurred $\$ 6,968$ higher cost than patients with T2DM without hemochromatosis, while having hemochromatosis secondary to hypertension, arthritis, CKD, or CLD resulted in $\$ 7,424$, $\$ 2,967, \$ 43,847$, and $\$ 16,634$, respectively, higher annual costs than hypertension, arthritis, CKD, or CLD patients without hemochromatosis.

A new hemochromatosis diagnosis was associated with an increase in both inpatient admissions and ED visits, a finding 
that is consistent with previous studies. ${ }^{23-25}$ In the case of either a new diagnosis or an existing one, our results show the excess in health care utilization and cost in hemochromatosis are mainly derived from an increase in outpatient visits and pharmaceutical claims. These findings may be explained by the need for phlebotomy sessions, blood work, and, occasionally, liver biopsies in managing hemochromatosis. ${ }^{26}$ Specifically, the cost of a phlebotomy session, performed in an outpatient setting, is approximately between $\$ 400$ and $\$ 1,400 . .^{25}$ Phlebotomy sessions have also been associated with increased side effects, which in turn can result in elevated health care utilization. ${ }^{27}$

Significant impact on health care costs associated with hemochromatosis was mainly seen as a function of higher CCI scores among those patients with diabetes and those with hypertension, arthritis, CKD, CLD, or heart failure. Increase in the number of comorbidities among hemochromatosis patients is essential in understanding the overall economic burden, since the majority of these patients are symptomatic (i.e., clinical penetrance is high). The associated higher costs in our study support a cross-sectional survey study done in Australia in the years 2013-2015, where the annual costs of hemochromatosis were dependent on disease severity, especially among those with the highest clinical penetrance. ${ }^{12}$

Abnormal iron hemostasis and iron overload have been cited as possible factors for increased risk of T2DM in hemochromatosis. ${ }^{28}$ In the multiracial, multiethnic Hemochromatosis and Iron Overload Screening (HEIRS) study of 97,470 participants in North America, 2\% of those who reported having T2DM also had hemochromatosis or iron overload. ${ }^{29}$ The presence of T2DM has also been associated with more severe hepatic fibrosis independent of iron loading, alcohol consumption, and male gender. ${ }^{11}$ This correlates with our finding of higher costs incurred in the group with both T2DM and hemochromatosis $(\$ 34,059)$ compared with those with T2DM but without hemochromatosis $(\$ 27,091)$. This finding suggests a potential benefit to screening patients with T2DM who are at increased risk of hemochromatosis.

Arthritis is an early, frequent, and severe symptom of hemochromatosis. In patients with hemochromatosis aged younger than 60 years, initial joint replacement surgeries have been noted to occur earlier in life, emphasizing the high musculoskeletal disease burden of the disease. ${ }^{30}$ A study including 2,851 patients with hemochromatosis concluded that symptoms had been present for an average of 10 years before the diagnosis was made..$^{31}$ In our study, \$25,109 was incurred per annum for patients with both arthritis and hemochromatosis compared with $\$ 22,141$ for those with just hemochromatosis.

Early signs of iron overload are ambiguous, and hemochromatosis penetrance is roughly $28.4 \%$ for men and $1.2 \%$ for women. ${ }^{6}$ Screening of those at risk has been suggested as a strategy to increase the rates of early detection in the precirrhotic stage, thereby reducing the clinical and economic burdens of hemochromatosis. ${ }^{9,14}$ A study of the cost-effectiveness of 165 hemochromatosis population screening algorithms suggested that early identification and subsequent treatment improves life expectancy from 68.6 years to 75.6 years for unscreened and screened patients, respectively. ${ }^{32}$ The same study also found that screening those aged 20-49 years was cost-effective, especially when penetrance is greater than $10 \%$. Our results support the findings of those studies, since the cost burden of hemochromatosis increases with age and is highest for patients aged 55 years or older.

\section{Limitations}

Our study is not without limitations. The MSCC database is based on ICD-9-CM codes, which might underestimate the prevalence of some comorbid conditions. Moreover, the disease diagnostic codes do not contain information on the severity of such comorbid conditions. The findings of this study may not represent the U.S. population, since the MSCC database only covers commercially insured subjects. The MSCC database did not allow for the evaluation of potential racial disparities in hemochromatosis health care utilization and economic burden. However, hemochromatosis is more common in the white nonHispanic population of the United States; therefore, the role of race/ethnicity is unlikely to have significant effects on our main findings.

This study also has several strengths. Our analysis included a large sample size (74,184 participants) and a matched control group to allow for the estimation of incremental differences in health care utilization and cost parameters. Utilization of a nested case-control study design within a cohort of insured subjects with continuous 12-month enrollment during the baseline period and for 12 months following the index date eliminates bias in selecting appropriate cases and controls. The use of propensity score matching ensured high comparability between cases and controls in relation to risk factors associated with hemochromatosis.

\section{Conclusions}

Our population-based analysis suggests that hemochromatosis imposes higher health care cost and utilization burdens compared with controls with the same demographics and comorbidity profiles. This burden is mostly a result of increased outpatient visits and pharmaceutical claims in hemochromatosis. A higher cost impact in hemochromatosis among those with additional comorbidities, especially those with T2DM, arthritis, CKD, or CLD, points to a need for earlier diagnosis to mitigate disease progression and health care cost burdens. Further research is needed to identify the cost burden of genotypic versus phenotypic hemochromatosis. 


\section{Authors}

MOHAMED I. ELSAID, MPH, ALM, LEED-GA; TINA JOHN, MD, MPH; YOU LI, BS; SOBHA KODURU, MD; SAIMA Z. ALI, MD; CAROLYN CATALANO, APN; and VINOD K. RUSTGI, MD, MBA, Department of Medicine, Division of Gastroenterology and Hepatology, Rutgers Robert Wood Johnson Medical School, New Brunswick, New Jersey. NAVANEETH NARAYANAN, PharmD, BCPS, Department of Pharmacy Practice, Ernest Mario School of Pharmacy, Rutgers The State University of New Jersey, Piscataway, and Division of Infectious Diseases, Rutgers Robert Wood Johnson Medical School, New Brunswick, New Jersey.

AUTHOR CORRESPONDENCE: Vinod K. Rustgi, MD, MBA, Rutgers Robert Wood Johnson Medical School, 1 Robert Wood Johnson Pl., Medical Educ. Bldg., Rm 466, New Brunswick, NJ 08901. Tel.: 301.801.5814; E-mail:vr262@rwjms.rutgers.edu.

\section{DISCLOSURES}

No outside funding supported this study. The authors have no relevant financial or other relationships to disclose.

An abstract containing some of the results from this study was accepted for the American Association for the Study of Liver Diseases Meeting; November 9-13, 2018; San Francisco, CA.

\section{ACKNOWLEDGMENTS}

The Rutgers University Ernest Mario School of Pharmacy provided the datasets analyzed in this study. The authors also thank Dr. Barbara de Graaff for providing her original thesis "The Health Economics of Haemochromatosis."

\section{REFERENCES}

1. Golfeyz S, Lewis S, Weisberg IS. Hemochromatosis: pathophysiology, evaluation, and management of hepatic iron overload with a focus on MRI. Expert Rev Gastroenterol Hepatol. 2018;12(8):767-78.

2. Wood MJ, Skoien R, Powell LW. The global burden of iron overload. Hepatol Int. 2009;3(3):434-44.

3. Crichton R. Iron Metabolism: From Molecular Mechanisms to Clinical Consequences. Chichester, UK: John Wiley \& Sons; 2016.

4. Pietrangelo A. Hereditary hemochromatosis: pathogenesis, diagnosis, and treatment. Gastroenterology. 2010;139(2):393-408.el-2.

5. Powell LW, Seckington RC, Deugnier Y. Haemochromatosis. Lancet. 2016;388(10045):706-16.

6. Allen KJ, Gurrin LC, Constantine CC, et al. Iron-overload-related disease in HFE hereditary hemochromatosis. N Engl J Med. 2008;358(3):221-30.

7. Adams P, Brissot P, Powell LW. EASL International Consensus Conference on Haemochromatosis: Part II. Expert Document. J Hepatol. 2000;33(3):487-96.

8. Adams PC, Barton JC. How I treat hemochromatosis. Blood. 2010;116(3):317-25.

9. Dubois S, Kowdley KV. The importance of screening for hemochromatosis. Arch Intern Med. 2003;163(20):2424-26; author reply 2426.

10. Shiani A, Narayanan S, Pena L, Friedman M. The role of diagnosis and treatment of underlying liver disease for the prognosis of primary liver cancer. Cancer Control. 2017;24(3):1073274817729240.

11. Wood MJ, Powell LW, Dixon JL, Ramm GA. Clinical cofactors and hepatic fibrosis in hereditary hemochromatosis: the role of diabetes mellitus. Hepatology. 2012;56(3):904-11.
12. de Graaff B, Neil A, Sanderson K, Yee KC, Palmer AJ. Costs associated with hereditary haemochromatosis in Australia: a cost-of-illness study. Aust Health Rev. 2017;41(3):254-67.

13. Nadakkavukaran IM, Gan EK, Olynyk JK. Screening for hereditary haemochromatosis. Pathology. 2012;44(2):148-52

14. de Graaff B, Neil A, Sanderson K, Si L, Yee KC, Palmer AJ. A systematic review and narrative synthesis of health economic studies conducted for hereditary haemochromatosis. Appl Health Econ Health Policy. 2015;13(5): 469-83.

15. de Graaff B, Neil A, Sanderson K, Yee KC, Palmer AJ. Quality of life utility values for hereditary haemochromatosis in Australia. Health Qual Life Outcomes. 2016;14:31.

16. Whitlock EP, Garlitz BA, Harris EL, Beil TL, Smith PR. Screening for hereditary hemochromatosis: a systematic review for the U.S. Preventive Services Task Force. Ann Intern Med. 2006;145(3):209-23.

17. Keehan SP, Cuckler GA, Sisko AM, et al. National health expenditure projections, 2014-24: spending growth faster than recent trends. Health Aff (Millwood). 2015;34(8):1407-17.

18. National Heart, Lung, and Blood Institute. Hemochromatosis. 2019. Available at: https://www.nhlbi.nih.gov/health-topics/hemochromatosis. Accessed November 4, 2019.

19. Quan H, Sundararajan V, Halfon P, et al. Coding algorithms for defining comorbidities in ICD-9-CM and ICD-10 administrative data. Med Care. 2005;43(11)1130-39.

20. Parsons LS. Performing a 1:N case-control match on propensity score. Poster presented at: Proceedings of the 29th Annual SAS Users Group International Conference; May 9-12, 2004; Montreal, Canada. Available at: https://support.sas.com/resources/papers/proceedings/proceedings/ sugi29/165-29.pdf. Accessed November 4, 2019.

21. Austin PC. Balance diagnostics for comparing the distribution of baseline covariates between treatment groups in propensity-score matched samples. Stat Med. 2009;28(25):3083-107.

22. Cohn J. Statistical power analysis for the behavioral sciences. Technometrics. 1988;31(4):499-500.

23. Brown AS, Gwinn M, Cogswell ME, Khoury MJ. Hemochromatosisassociated morbidity in the United States: an analysis of the National Hospital Discharge Survey, 1979-1997. Genet Med. 2001;3(2):109-11.

24. Cowan M, Westlake S, Thomson S, et al. The increasing hospital disease burden of haemochromatosis in England. Aliment Pharmacol Ther. 2010;31(2):247-52.

25. America Hemochromatosis Society. FAQ's about hemochromatosis/ iron overload. Available at: https://www.americanhs.org/faq.htm. Accessed November 4, 2019.

26. Bacon BR, Adams PC, Kowdley KV, Powell LW, Tavill AS; American Association for the Study of Liver Diseases. Diagnosis and management of hemochromatosis: 2011 practice guideline by the American Association for the Study of Liver Diseases. Hepatology. 2011;54(1):328-43.

27. Brissot P, Ball S, Rofail D, Cannon H, Jin VW. Hereditary hemochromatosis: patient experiences of the disease and phlebotomy treatment. Transfusion. 2011;51(6):1331-38.

28. Barton JC, Acton RT. Diabetes in HFE hemochromatosis. J Diabetes Res. 2017;2017:9826930. Available at: https://www.ncbi.nlm.nih.gov/pmc/ articles/PMC5346371/. Accessed November 4, 2019.

29. Acton RT, Barton JC, Passmore LV, et al. Relationships of serum ferritin, transferrin saturation, and HFE mutations and self-reported diabetes in the Hemochromatosis and Iron Overload Screening (HEIRS) study. Diabetes Care. 2006;29(9):2084-89.

30. Sahinbegovic E, Dallos T, Aigner E, et al. Musculoskeletal disease burden of hereditary hemochromatosis. Arthritis Rheum. 2010;62(12):3792-98. 
31. McDonnell SM, Preston BL, Jewell SA, et al. A survey of 2,851 patients with hemochromatosis: symptoms and response to treatment. Am J Med. 1999;106(6):619-24.
32. Gagne G, Reinharz D, Laflamme N, Adams PC, Rousseau F. Hereditary hemochromatosis screening: effect of mutation penetrance and prevalence on cost-effectiveness of testing algorithms. Clin Genet. 2007;71(1):46-58.

\begin{tabular}{|c|c|c|c|c|}
\hline \multirow{2}{*}{$\begin{array}{l}\text { APPENDIX } \\
\text { Health Care Cost } \\
\end{array}$} & \multicolumn{4}{|c|}{$\begin{array}{l}\text { Annual Per-Patient Health Care Costs in the } 12 \text { Months Following Index Date by Comorbidity } \\
\text { Status for Matched Pairs of Hemochromatosis Cases and Chronic Liver Disease Controls Without } \\
\text { Hemochromatosis, 2011-2014a }\end{array}$} \\
\hline & Number of Pairs & $\begin{array}{c}\text { Hemochromatosis } \\
\mathbf{n}=37,092 \\
\$(95 \% \mathrm{CI})\end{array}$ & $\begin{array}{c}\text { No Hemochromatosis } \\
\mathbf{n}=37,092 \\
\$(95 \% \mathrm{CI})\end{array}$ & $P$ Value ${ }^{b}$ \\
\hline \multicolumn{5}{|c|}{ Charlson Comorbidity Index score group } \\
\hline 0 & 24,228 & $(10,999-11,823)$ & $(11,319-12,055)$ & 0.5836 \\
\hline 1 & 6,889 & $(17,459-19,244)$ & $(17,052-18,753)$ & 0.0578 \\
\hline 2 & 2,713 & $(32,274-38,907)$ & $(26,660-32,153)$ & 0.0019 \\
\hline 3 & 1,327 & $(38,416-50,793)$ & $(29,140-35,414)$ & 0.0142 \\
\hline $4+$ & 1,463 & $(97,116-114,147)$ & $(67,906-80,567)$ & $<0.0001$ \\
\hline Type 2 diabetes & 5,296 & $(31,902-36,217)$ & $(25,434-28,748)$ & $<0.0001$ \\
\hline Hypertension & 6,994 & $(30,603-34,391)$ & $(23,760-26,386)$ & $<0.0001$ \\
\hline Chronic liver disease & 4,560 & $(36,977-43,144)$ & $(21,843-25,010)$ & $<0.0001$ \\
\hline Chronic kidney disease & 169 & $149,747 \quad(120,022-179,471)$ & $(73,447-138,352)$ & $<0.0001$ \\
\hline Alcohol abuse or dependence & 145 & $(34,059-65,788)$ & $(27,527-43,662)$ & 0.2582 \\
\hline Arthritis & 15,241 & $(24,129-26,088)$ & $(21,343-22,940)$ & 0.0002 \\
\hline Heart failure & 135 & $(88,135-154,313)$ & $(47,111-87,242)$ & 0.0002 \\
\hline Myocardial infarction & 198 & $(53,324-89,509)$ & $(40,883-75,059)$ & 0.4125 \\
\hline \multicolumn{5}{|c|}{$\begin{array}{l}\text { a All costs were adjusted to } 2017 \text { U.S. dollars using the medical care component of the Consumer Price Index. } \\
\text { b Obtained from Wilcoxon signed rank tests for cost comparisons between cases versus controls of the same patient subgroup in the } 12 \text { months following index date } \\
\text { (first diagnosis date of hemochromatosis for cases and a randomly selected date from all claim records for controls). } \\
\text { CI=confidence intervals. }\end{array}$} \\
\hline
\end{tabular}

